\title{
ANAESTHETIC MANAGEMENT FOR INTERVENTIONAL NEURORADIOLOGY
}

\section{Dr Shirani Hapuarachchi}

Interventional neuroradiology dates back to the year 1930 when for the first time occlusion of blood vessels by an endo vascular approach was first described for the treatment of a traumatic arterio-venous fistula. Due to the limitations in catheter technology and imaging there was no further development till the 1960s when the first endovascular approach to treat a cerebral arterio venous malformation was carried out.

The 1970s saw a rapid development of imaging, the invention of the Digital subtraction angiography machine which was of immense help in visualizing small vessels without the interference of bony structures. This is the first step in Road mapping of the vessel which involves projecting a subtracted vascular pathway so that the blood vessel is continuously displayed. This allows the movement of the catheter to be followed along the blood vessel.

The development of catheters, and guide wires all paved the way for successful treatment of AV malformations and aneurysms by this new technique. Both cranial as well as spinal neuro vascular conditions are treated by this method.

The other procedures carried out by this method are preoperative embolisation of vascular tumours mainly highly vascular meningiomas.

The types of embolisations carried out are coil embolisations for aneurysms, embolisation using onyx or cyanoacrylate for arterio-venous malformations.

Coils are made of Platinum. They are attached to an electronically detachable pusher wire and introduced via a micro catheter from which it is detached once in place. Several coils may be necessary to occlude an aneurysm.

Onyx is an embolising agent used for arterio venous malformations. It consists of a vinyl alcohol co polymer dissolved in dimethyl sulphoxide. Tantalum powder is added as contrast for fluoroscopy. The advantage is that it precipates immediately but is non adhesive unlike cyanoacrylate so that controlled injection and filling of the nidus can take over several minutes. This facilitates time for angiography. It also does not liberate heat. It can reach difficult locations and can penetrate large number of feeder vessels in one injection.

Digital Subtraction Angiography was first introduced to the Cardiology unit of the National Hospital in Sri Lanka in the year 1995. Aneurysms at that time were diagnosed by direct carotid angiography and only selected patients were sent for digital subtraction angiography to the cardiology unit. These were patients whose angiograms did not give a clear picture which was essential for definitive care which was surgical clipping of the aneurysm.

Complications were frequent and these ranged from seizures to loss of conciousness and even cardiac arrest. There were also patients who developed reactions to the contrast. At that time we did not have non ionic contrast media. With the availability of non ionic contrast media OMNIPAQUE this problem became non existent.

With the availability of a new Digital subtraction angiography fluoroscopy machine purchased in 2000 we were able to do the DSAs in the National Hospital of Sri Lanka. With this facility being 
available, the next step was the development of invasive endovascular neuro radiological procedures.

In Sri Lanka the first case that we did was in the year 2004 for an aneurysm which was successfully coiled by a visiting Indian Neuro radiologist for which our Neuro anaesthetic team successfully administered the anaesthetic. Thereafter until the year 2007 the local radiological team assisted by the Indian team carried out the interventions. From the year 2007 the local team headed by consultant interventional radiologist Dr. Nihal Wijewardene have been carrying out the procedures. For all these interventions it was our local anaesthetic team who successfully administered anaesthesia.

Up to now we have carried out a total of 24 endovascular procedures. Of them 15 were males and 09 were females. The ages ranged from 8 to 50 years. We had 2 patients in the 0-8 range, 07 patients in the 10-20 range, 09 patients in the $20-$ 30 range, 04 patients in the $30-40$ range and 02 patients in the $40-50$ range.

The pre operative GCS on admission as an emergency ranged from 3-15 but was $14-15$ at the time of taking in for the procedure in all except one who had a GCS of 7.

Of the 22 patients, 17 patients had AVM's and 04 patients had aneurysms. One was a spinal arterio venous malformation.

14 patients had embolisations with onyx and 07 patients had coil embolisations, one had cyano acrylate glue instead of onyx. One of the coils inserted were an electronically detachable GDC coil.

\section{Results}

10 patients had $100 \%$ occlusion while 3 patients had $90 \%$ occlusion and the rest had $50 \%$ occlusion. The reason for mentioning the extent of occlusion is that these patients will be reassessed again months later and may have the procedure repeated under GA again.

There was one death following massive intracerebral haemorrhage post operatively. This was a patient with a GCS of 7 preoperatively and was considered very high risk.

There was one patient who developed a subarachnoid haemorrhage intraoperatively, but recovered completely.

\section{Anaesthetic Management}

Patients present with headache, vomiting, seizures, drowsiness, loss of conciousness, cranial nerve palsies, and focal neurological deficits.

Once a clinical diagnosis of aneurysm is made the patient is managed in the intensive care unit if a bed is available with strict bed rest, nimodipine is given to prevent vaso spasm, blood pressures are maintained at normal levels to prevent a re-bleed while at the same time preventing ischaemia of the brain.

In patients with AVMs the clinical presentation is the same. Following the clinical diagnosis a CT scan is carried out which may either show a subarachnoid haemorrhage in a patient with an aneurysmal rupture or an intracerebral haematoma in a ruptured arterio venous malformation. Thereafter a digital subtraction angiography is done for a definitive diagnosis. This is carried out under sedation with fentanyl and propofol or midazolam infusions depending on the patients pre operative Glasgow coma score.

Once a definitive diagnosis is made the patient is taken up for the endovascular occlusion by embolisation with onyx in the case of an arterio venous malformation or coil embolisation in the event of an aneurysm.

\section{Pre operative assessment}

It is imperative that a detailed preoperative assessment should be carried out in all these patients similar to any other surgical intervention. This is usually carried out in the neuro surgical ward as most patients are inward patients having been admitted as neuro surgical emergencies. It is also essential to know the diagnosis so that the surgical needs are met in the most appropriate manner. Pre operative neurological deficits too should be carefully assessed clinically as well as radiologically as this may not only influence our management but may be important when assessing post operative recovery. Special emphasis is placed on cerebral perfusion and detailed coagulation profile. It is very important to obtain a history of allergy as contrast medium is used. Premedication is generally not advocated. Unless the patient gives a history of allergy in which situation the patient is premedicated with steroids 
- Hydrocortisone and promethazine. Patients who give a history of seizures are prescribed Dilantin sodium.

A general anaesthetic is the method of choice. The initial DSA is carried out while the patient is awake therefore the patients is told about the hot flushing he may experience with injection of the dye.

\section{Anaesthetic Management}

The procedure is carried out in the DSA room in the Radiological suite of the National Hospital which is away from all other theatres and therefore in a sense "isolated"

The DSA room has been prepared with its own Anaesthetic machine, multi parameter monitor which has facilities for electrocardiography, non invasive blood pressure, pulse oximetry to continuously detect oxygen saturation and capnography. The monitor should have a very good display with large digits for values and contrasting colour for tracings as the room is darkened and the anaesthetist should be able to read the parameters from the console area. It also has syringe pumps, infusion lines with octopus, anaesthetic ventilator, ACT machine for serial measurement of activated clotting time, and the fluoroscopy machine. So you can see the amount of equipment that is absolutely necessary to carry out such procedures.

In addition you need adequate space for the radiological team to work and for movement of the fluoroscopy machine and the table. The Anaesthetic machine is kept near the patients feet and on the opposite side to that of the working party. Therefore long tubes with adequate slack is a necessity. The infusion lines and monitoring lines too should be long with adequate slack so that there is no hindrance to table movement and fluoroscopy machine movement.

\section{Environmental pollution}

Radiation is one of the biggest problems faced by the team. There are three sources of radiation in the DSA room. One is directly from the fluoroscopy machine, the second is leakage radiation through protective barriers and the third comes from scatter radiation from the patient. The anaesthetic team does not remain in the DSA room when not needed. They observe the patient from the adjoining console area. Still they wear lead aprons and thyroid shields as they may have to be in the room at the beginning when the first DSA is done and also may have to enter the room in the event of a critical incident. To minimize this problem the patient once anaesthetized is maintained on infusions with extension tubings and the anaesthetic machine too is kept well away from the head end and on the opposite side of the work area.

\section{Anaesthesia}

Once the patient arrives in the DSA room and the identity checked and the patient is taken over by the nurse in the DSA room the patient is transferred to the table and intravascular access is established. A $500 \mathrm{ml}$ bag of $0.9 \%$ normal saline is infused and baseline monitoring established.

Thereafter the initial DSA is done by the radiology team in order to obtain the road map which I described to you earlier. The patient is awake. Arterial cannulation is carried out following local anaesthetic infiltration on the Right Femoral artery as the left side is reserved for the anaesthetist to establish left femoral vein access. In two patients brachial artery was used. One of our patients who had a preoperative GCS of 7 was already intubated and ventilated in the ICU and continued to be so during the initial DSA. All other patients as mentioned earlier had a GCS of $14-15$ and was awake during the initial DSA.

Following local anaesthetic infiltration a sheath is introduced into the artery which is usually 6.0 French gauge. This acts as an introducer to a 4-6 FG catheter which is guided into the carotid or vertebral arteries under fluoroscopic control. This acts as a guide to a supraselective smaller catheter into the cerebral circulation. It is via this catheter that coils or embolic agents are introduced.

\section{Anticoagulation}

First a base line ACT is carried out. Thereafter a loading dose of $70 \mathrm{IU} / \mathrm{kg}$ heparin is given followed by $100 \mathrm{IU} /$ hour to prolong ACT by 2-3 times the normal. Hourly ACT is done so that adequate anticoagulation is present throughout the procedure. 


\section{Anaesthetic Procedure}

As I mentioned before following the initial DSA the patient is now anaesthetized. A general anaesthetic is preferred as the patient needs to be very still during the procedure. An immobile patient is a necessity if clear images are to be obtained. It also gives the anaesthetist better control of the patients cardiovascular and respiratory parameters during anticoagulation and embolisation.

\section{Method}

The patient is anaesthetized with fentanyl 2 micrograms $/ \mathrm{kg}$ IV followed by $2 \mathrm{mg} / \mathrm{kg}$ of Propofol and midazolam $0.1 \mathrm{mg} / \mathrm{kg}$ IV which is followed by atracurium $0.5 \mathrm{mg} / \mathrm{kg}$ or vecuronium $0.1 \mathrm{mg} / \mathrm{kg}$. The patient is intubated with a reinforced tracheal tube of appropriate size and therafter connected to the ventilator.

The patient is connected to IV infusions of propofol $(4 \mathrm{mg} / \mathrm{kg} / \mathrm{hour})$ muscle relaxant and fentanyl. $0.5 \%$ of Isoflurane too is added. Nitrous oxide is avoided as this can give rise to expansion of micro air bubbles during injection of contrast and during irrigation.

\section{Blood Pressure control}

During coil embolisation of aneurysms the blood pressure is kept normal during the procedure while in patients with AVM the blood pressures are dropped by controlled hypotension to reduce the flow across the AVM. This is by means of the propofol infusion, where the rate is increased.

The left femoral access is used for blood withdrawal from one port, one port is used for heparin injection and the anaesthetic infusions are connected via the octopus.

At the end of the procedure the sheath is kept in situ while the catheters are removed. The heparin is reversed totally with protamine. $1 \mathrm{mg}$ of protamine for each 100 units of heparin is given. The patient is transferred to the ICU still anaesthetized and ventilated. Once settled in the ICU the muscle relaxants and fentanyl are stopped, the patient weaned off from the ventilator and once adequate spontaneous respiration is established the propofol is stopped and the patient allowed to wake up. The ACT is repeated every 4hours until it is normal. At which point the sheath is removed and the patient sent to the ward. While the sheath is in situ the patient needs to lie supine.

During the stay in the ICU the patient is monitored neurologically by checking the level of consciousness with the GCS score, pupils, the CVS with heart rate, blood pressure and urine output. The latter is very necessary as contrast has been given. Meticulous fluid balance charts are essential due to the diuresis following injection of contrast. In patients with coil embolisation of aneurysms the blood pressures are kept as near normal as possible. In patients with AVMs the arterial pressures are kept $15-20 \%$ below the patient's normal level. The respiration is monitored by measuring the tidal volume, rate and airway pressure initially while the patient is on the ventilator, the oxygen saturation and arterial blood gases. If embolic complications are expected then anticoagulation is carried out for a further 24 hours. All patients were given metoclopromide 10mg 8hourly to avoid nausea and vomiting. Nimodipine was continued in patients with vasospasm via naso gastric tube.

\section{Complications}

We had one patient as I mentioned earlier who had a preoperative GCS of 7 re bleeding during the procedure and the patient was continuously ventilated post op and died on the $3^{\text {rd }}$ post op day.

A second complication was a very unfortunate incident when the catheter was being removed the patient developed a subarachnoid haemorrhage. The Heparin was immediately reversed completely with protamine while monitoring the ACT. The patient was managed successfully with continous ventilation and sedation without waking up the patient for 48 hours after which we repeated the CT which showed very little blood in the subarachnoid space. Thereafter the patient was weaned off and recovered completely with a post op GCS of 15 and no residual neurological effects. The patient was discharged home without any neurological deficits.

These were the only two complications we had in the 22 patients done so far. With good skills and a good working environment the risk is very low when compared to the risk of surgery and non intervention. 
If no intervention is carried out for a ruptured aneurysm the risk of a re bleed is $4 \%$ in the first 24 hours and a $1 \%$ risk per day thereafter. So death becomes inevitable with time.

The success of the aneurysm depends on the neck diameter and the size of the body of the aneurysm. With a neck diameter of $4 \mathrm{~mm}$ and less the success rate is high whilst with neck diameters more than $4 \mathrm{~mm}$ the success rate is low. In our 4 patients the neck diameter was less than $4 \mathrm{~mm}$. When the body is large a large number of coils will be necessary and also the incidence of post canalization is also high. The patient who died had a giant aneurysm.

With AVMs the risk of haemorrhage after diagnosis is $2-4 \%$ per year. With large AVMs embolisation is sometimes carried out to reduce the size of the nidus before resection.

Majority of our patients are arteriovonous malformations. 18 out of 22 . Only one of them had an intraoperative complication but survived without any residual effects.

We have had $100 \%$ success in the patients who survived post operatively so far. They are being assessed in the neuro surgical clinics following discharge. They will be assessed after 6 months with a repeat angiogram, This is where a MRA becomes useful as it is non invasive but is not available to us. Another problem is recanalisation of the coiled aneurysm. This is particularly seen with wide neck and giant aneurysms.
This is a short series of 22 patients. It is not because aneurysms and AVMs are a rarity in our country but it is because of the cost involved. A single coil is Rs 100,000/= and many coils are needed for a single patient. Also until we start the procedure it is very difficult to predict the number of coils that will be needed. We have used 2- 10 coils in our patients. Therefore you can see that the cost of the coils could go up to a million rupees. We have been getting some assistance from the Medical Supplies division but it is not possible on a regular basis.

Therefore we still continue with surgical resection of AVMs and surgical clipping of aneurysms on a larger scale almost on a daily basis.

The main advantage in coil embolisation is that hospital stay is short with only one day in ICU and another day in the ward as opposed to surgical intervention where the patient stays at least 3 days in ICU and at least 1 week in the ward.

The patient compliance is also very high with interventional neuro radiological procedures.

At present we carry out digital subtraction angiography within 24 hours of patient admission, in the future our aim is to carry out interventional neuro radiological procedures too as soon as a diagnosis of aneurysm or AVM is made in the emergency department. 